\title{
EXPERIÊNCIAS COM MODELAGEM MATEMÁTICA EM UM CURSO DE LICENCIATURA EM MATEMÁTICA NA MODALIDADE DE EDUCAÇÃO A DISTÂNCIA
}

\author{
LONDRINA/PR JULHO/2018
}

\author{
Daiany Cristiny Ramos - UNOPAR - daiany.ramos@unopar.br \\ Diego Fogaça Carvalho - UNOPAR - diego.fogaca@kroton.com.br \\ Alessandra Negrini Dalla Barba - UNOPAR - alessandra.barba@kroton.com.br \\ Hallynnee Héllenn Pires Rossetto - UNOPAR - hallynnee.rossetto@kroton.com.br \\ Jenai Oliveira Cazetta - UNOPAR - cazetta.jenai@gmail.com \\ Renata Karoline Fernandes - UNOPAR - renata.karoline@kroton.com.br
}

Tipo: Relato de Experiência Inovadora (EI)

Categoria: Conteúdos e Habilidades

Setor Educacional: EDUCAÇÃO SUPERIOR

\begin{abstract}
RESUMO
Este trabalho tem por objetivo apresentar as práticas de modelagem matemática desenvolvidas no curso de Licenciatura em Matemática, na modalidade de Educação a Distância, da Universidade Pitágoras Unopar - UNOPAR, principalmente no ano de 2017, por meio da realização de um trabalho acadêmico, denominado produções textuais. A modelagem matemática é compreendida como uma alternativa pedagógica que aborda problemas não essencialmente matemáticos, por meio da Matemática. Pautados nessa concepção, a inserção das atividades é iniciada na disciplina de Seminários da Prática, que compõe a grade curricular do quinto semestre do curso. A intenção de utilizar a Modelagem Matemática nas aulas e, principalmente, nas produções textuais, consiste em desenvolver atividades que permitam que os estudantes utilizem diferentes linguagens matemáticas, organizem e representem os dados, bem como desenvolvam e interpretem modelos matemáticos que tenham potencial para descrever situações do cotidiano.
\end{abstract}

Palavras-chave: Modelagem Matemática. Educação Matemática. Ensino a distância.

\section{AGRADECIMENTOS}

AGRADECEMOS À EQUIPE DE DOCENTES DO CURSO DE LICENCIATURA EM MATEMÁTICA DA UNOPAR E ANHANGUERA. 


\section{Introdução}

Um dos objetivos do curso de Licenciatura em Matemática consiste em se tornar um ambiente de formação inicial de professores em que vários conhecimentos a respeito da prática pedagógica sejam construídos. Dentre a pluralidade desses conhecimentos, que inclui o conteúdo matemático, a Educação Matemática, a didática e os saberes relacionados à prática docente, pode-se destacar o estudo da modelagem matemática enquanto alternativa pedagógica, que permite uma leitura e interpretação parcial e idiossincrática de uma situação do cotidiano por meio da Matemática.

A modelagem matemática, apesar de ter sido desenvolvida na área da Matemática Aplicada, sofreu diversas influências até tornar-se um tema de estudo da Educação Matemática. Nesse sentido, diversas perspectivas foram desenvolvidas, de modo a analisar as contribuições da modelagem para o ensino de Matemática.

Adotando a perspectiva da modelagem matemática enquanto alternativa pedagógica, são realizados diversos trabalhos voltados ao estudo dessa tendência, como, por exemplo, sua presença enquanto disciplina na matriz curricular, além da realização de estudos na disciplina de Seminário da Prática e a proposição de trabalhos desenvolvidos de forma colaborativa, envolvendo atividades de modelagem. $\mathrm{Na}$ sequência, são apresentadas as características dos trabalhos desenvolvidos na graduação a distância em relação à modelagem matemática e suas potencialidades para o ensino da Matemática.

\section{Objetivo do trabalho}

Este relato tem a intenção de apresentar a experimentação de condução e o desenvolvimento de práticas de modelagem matemática aplicadas no curso de Licenciatura em Matemática, na modalidade Educação a Distância, da Universidade Pitágoras Unopar - UNOPAR, principalmente no ano de 2017, por meio da realização de produções textuais.

\section{Procedimentos Metodológicos}

Este trabalho é composto por um relato de experiência a respeito das práticas de modelagem matemática desenvolvidas no curso de Licenciatura em Matemática na Universidade Pitágoras Unopar - UNOPAR, sendo predominantemente qualitativo, descritivo e exploratório. 
No que diz respeito à característica descritiva, autores afirmam que esta apresenta inferência direta a respeito do objeto que está sendo analisado, no caso, as práticas de modelagem matemática. A fundamentação do relato pautou-se, ainda, em procedimentos característicos de estudo de caso que, para Goog e Halt (1979), tem como característica a compreensão de situações que podem ou não ser generalizadas, tendo como princípio o levantamento e a organização de dados, preservando o objeto estudado.

\section{Modelagem Matemática na Educação Matemática}

A modelagem matemática é um método originalmente voltado para a construção de modelos na Matemática Aplicada, e quando usada em sala de aula, pode ajudar o estudante a entender a Matemática por meio de temas do cotidiano.

Segundo Meyer, Caldeira e Malheiros (2011), a modelagem matemática apresenta várias definições na Educação e são pequenas sutilezas que as tornam diferentes. Para Bassanezi (2011), a modelagem matemática é considerada como um método de investigação que pode criar uma relação entre a Matemática e outras áreas do conhecimento. Por outro lado, Burak (1987) a considera como uma metodologia de ensino que visa explicar situações do cotidiano matematicamente. Borba, Meneguetti e Hermini (1997), consideram-na como uma estratégia pedagógica, em que os estudantes são responsáveis pela escolha do tema a ser estudado. Nessa perspectiva, a modelagem é concebida com características interdisciplinares, pois os problemas são abertos e não se restringem à disciplina de Matemática.

Por serem várias as definições para a modelagem matemática, faz-se necessário adotar uma delas na sala de aula e na pesquisa, pois essa definição mostra qual o caminho que o professor ou o pesquisador vai seguir, já que cada perspectiva tem suas peculiaridades e sutilezas. Entendemos a modelagem matemática como uma alternativa pedagógica que aborda, por meio da Matemática, situações não essencialmente matemáticas (ALMEIDA e DIAS, 2005). Nessa perspectiva, a modelagem matemática pode viabilizar uma leitura e interpretação parcial dos fenômenos em estudo, sendo feita por meio de uma representação denominada modelo matemático e que constitui "um sistema conceitual, descritivo ou explicativo, expresso por meio de uma linguagem ou estrutura matemática e tem por finalidade descrever ou explicar o comportamento de outro sistema" (ALMEIDA; SILVA; VERTUAN, 2012, p. 13).

Almeida e Vertuan (2014) sugerem que a prática de atividades de modelagem permite uma familiarização tanto dos alunos quanto dos professores com atividades com essas 
características. Tal familiarização pode ajudar na implementação da modelagem matemática em sala de aula e na superação de alguns obstáculos enfrentados pelos alunos e pelos professores. Nesse contexto, Almeida, Silva e Vertuan (2012, p.26) sugerem que a modelagem seja introduzida em sala em três momentos, da seguinte forma:

Em um primeiro momento, o professor coloca os alunos em contato com uma situaçãoproblema, juntamente com os dados e as informações necessárias. A investigação do problema, a dedução, a análise e a utilização de um modelo matemático são acompanhadas pelo professor, de modo que ações como definição de variáveis e hipótese, a simplificação, a transição para a linguagem matemática, obtenção e validação do modelo bem como o seu uso para análise da situação, são em certa medida, orientadas e avaliadas pelo professor. Posteriormente, em um segundo momento, uma situação-problema é sugerida pelo professor aos alunos e esses, divididos em grupos, complementam a coleta de informações para a investigação da situação e realizam a definição de variáveis e a formulação de hipóteses simplificadoras, a obtenção e validação do modelo matemático e seu uso para a análise da situação. $O$ que muda, essencialmente, do primeiro momento para o segundo é a independência do estudante o que se refere à definição de procedimentos extra matemáticos e matemáticos adequados para a realização da investigação. Finalmente, no terceiro momento, os alunos divididos em grupos, são responsáveis pela condução de uma atividade de modelagem, cabendo a eles a identificação de uma situação-problema, a coleta e análise dos dados, as transições de linguagem, a identificação de conceitos matemáticos, a obtenção e validação do modelo e seu uso para a análise da situação, bem como a comunicação desta investigação para a comunidade escolar.

As experiências vivenciadas nos dois primeiros momentos permitem que o aluno adquira confiança e autonomia para desenvolver, por si só, uma atividade, ou seja, vivenciar o terceiro momento. Os dois primeiros momentos ajudam o aluno a entender como é o desenvolvimento de uma atividade de modelagem, fazendo que eles se sintam menos perdidos em atividade futuras.

Diante do exposto, apresentaremos, a seguir, como a disciplina de Modelagem Matemática, para o curso de Licenciatura em Matemática, na modalidade de Educação a Distância, foi estruturada.

\section{A disciplina de Modelagem Matemática no curso de Matemática da UNOPAR - EaD}

A disciplina de Modelagem Matemática, no curso de Licenciatura em Matemática, 
compõe a grade curricular, sendo uma disciplina obrigatória do sexto semestre. Por se tratar de curso na modalidade de Educação a Distância, a disciplina é organizada em teleaulas, aulas-atividades e discussões no fórum, o qual está disponível no ambiente virtual de aprendizagem durante toda a disciplina.

No segundo semestre de 2017, a disciplina foi organizada de modo a contemplar quatro teleaulas, que correspondem a um dos momentos síncronos da disciplina, no qual são ministradas aulas transmitidas em tempo real aos alunos e que foram preparadas visando abordar aspectos da modelagem matemática, bem como atividades de modelagem matemática. Em conjunto com as teleaulas há um momento em que os estudantes realizam atividades que, na disciplina em questão, tinham por objetivo explorar os conceitos vistos em teleaula por meio da realização de estudos teóricos e/ou o desenvolvimento de tarefas de modelagem propriamente ditas. As teleaulas foram organizadas para discutir três questões: "O que é modelagem matemática?", "Como fazer modelagem matemática?" e "Por que utilizar modelagem matemática?". Em um primeiro momento, os estudantes tiveram contato com a modelagem matemática na perspectiva da Matemática Aplicada por meio dos modelos populacionais de Malthus e Verhulst (BASSANEZI, 2011). Nesse momento, foram apresentados os modelos prontos para que os estudantes conhecessem suas principais características, porém, durante essa aula, os estudantes manifestaram interesse em conhecer a dedução desses modelos matemáticos, o que possibilitou a realização de uma aula adicional sobre a dedução dos modelos de Malthus e Verhulst.

Em um segundo momento, os estudantes tiveram contato com a modelagem matemática no âmbito da Educação Matemática. Foram apresentadas algumas perspectivas da modelagem matemática e destacou-se a importância de se assumir uma única perspectiva que orientará as atividades.

Foram desenvolvidas duas atividades, em um primeiro momento, com os alunos durante as teleaulas: Casa própria: será que com o salário dá? e O horário de verão. Nas atividades, o docente responsável conduziu todo o seu desenvolvimento, fazendo questionamentos durante a aula de modo a incluir o estudante no desenvolvimento da atividade. A atividade $O$ horário de verão foi desenvolvida com o auxílio do software GeoGebra visando estudar de forma algébrica e geométrica a função trigonométrica cosseno com seus parâmetros. $O$ desenvolvimento dessas atividades oportunizou aos estudantes o contato com as etapas de uma atividade de modelagem matemática, bem como destacou que a modelagem matemática, no âmbito da Educação Matemática, vai além da construção de um modelo matemático. 
A disciplina de Modelagem Matemática não é o único momento em que os estudantes têm contato com a modelagem, pois os trabalhos com atividades, segundo essa perspectiva, têm início na disciplina de Seminário da Prática $\mathrm{V}$, referente ao 5o período e, posteriormente, em algumas das Produções Textuais em Grupo desenvolvidas a cada semestre pelos estudantes. Na próxima seção, discutiremos sobre essas atividades.

\section{As atividades de Modelagem Matemática desenvolvidas no curso}

No ano de 2017, uma das teleaulas e aulas atividades de uma disciplina abordou o tema da Modelagem Matemática, porém sem, inicialmente, defini-la como uma atividade de modelagem. Nesse momento, a atividade desenvolvida se denominava $O$ desafio do balde de gelo. A partir desse tema, e antes da realização das atividades, alguns estudantes comentaram que, em um primeiro momento, acreditavam que seria desenvolvida uma atividade lúdica e não uma atividade que envolvesse matemática. $O$ objetivo da aula era estudar a situação-problema do desafio do balde de gelo, propagada pelas redes sociais na Internet, utilizando os conceitos de progressão geométrica e, ao final da teleaula, foram apresentadas, aos estudantes, as características de uma atividade de modelagem de modo a caracterizar a atividade desenvolvida como tal. É importante destacar que, durante todo o desenvolvimento, os alunos eram questionados sobre aspectos da situação e formas de resolução.

No 6º período do curso, além da disciplina de Modelagem Matemática, os alunos desenvolvem atividade de modelagem matemática em grupos, caracterizadas como atividades do segundo momento, visto que são propostas situações-problemas e problemas a serem investigados pelos estudantes. Esse trabalho corresponde a uma das tarefas que os alunos precisam desenvolver a cada período do curso e que são denominadas Produções Textuais em Grupo (PTGs). Assim, ao longo do semestre, principalmente no $6^{\circ}$ período, os estudantes precisam desenvolver uma atividade de modelagem matemática em grupos, envolvendo os conhecimentos abordados nas diferentes disciplinas estudadas ao longo do período em questão.

Nos dois últimos anos, os estudantes do 6ำ período já desenvolveram duas atividades desse tipo com temáticas diferenciadas. No ano de 2016, a proposta desenvolvida apresentou como tema a Venda de veículos no Brasil, cuja situação-problema estava relacionada ao número de emplacamentos de carros no Brasil. Com base em dados atualizados em relação aos emplacamentos realizados no período de 2006 a 2015, no país, os estudantes deveriam construir um modelo matemático que representasse 0 volume de emplacamentos de veículos. Além disso, deveriam analisar o modelo com base na situação em estudo e relacionando-o aos conceitos estudados na disciplina de 
Cálculo Diferencial e Integral, cursada por eles no período considerado. Conforme a Figura 1(a), apresentada a seguir, o grupo G1 construiu um gráfico para analisar graficamente o comportamento dos dados que representam a situação em estudo. Outros grupos também adotaram esse processo para a construção do modelo, sendo que alguns identificaram que as melhores representações seriam por meio de funções polinomiais de $2^{\circ}$ grau, ou ainda por funções definidas por partes associadas a diferentes funções polinomiais de $1^{\circ}$ grau.

Figura 1 - Estudo do conjunto de dados correspondentes às PTGs.

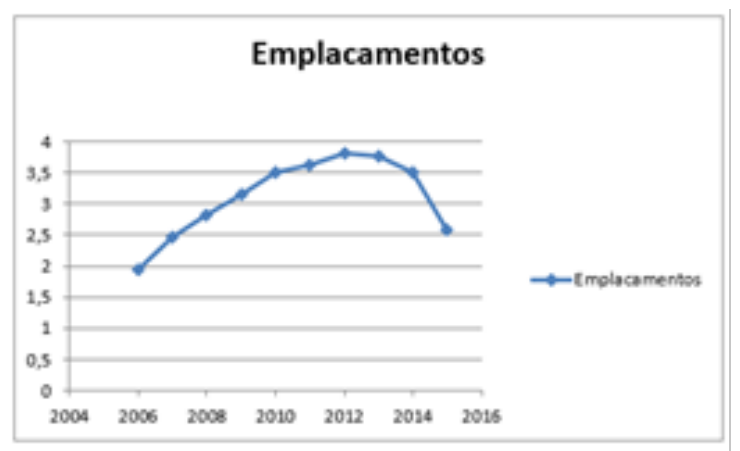

(a) Dailus daProdicto Textmal emGrupo de 2016

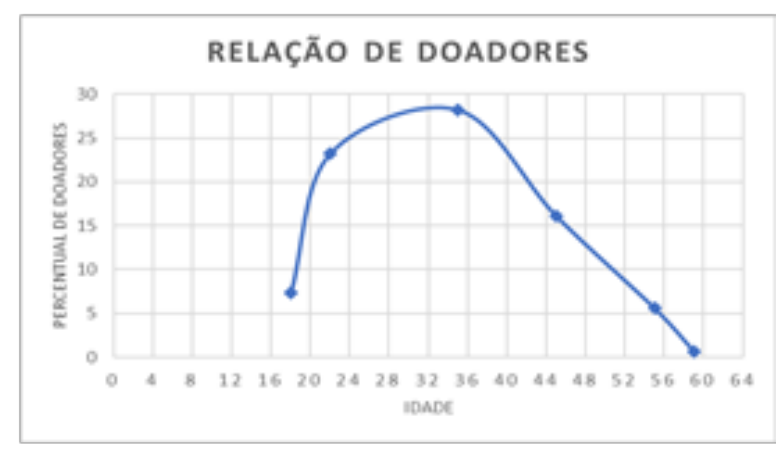

(b) Daidos daProingâno Textinal emGrupo de 2017

Fonte: Registro dos alunos

Em 2016 os alunos foram mobilizados a refletir sobre a aplicação das atividades por eles elaboradas na Educação Básica, enfatizando a viabilidade da aplicação das atividades de modelagem matemática, na perspectiva adotada nesse trabalho, para a aprendizagem da Matemática nos anos finais do Ensino Fundamental e/ou Ensino Médio. No trecho a seguir, destacamos as reflexões apresentadas pelo grupo G2 a respeito da importância da modelagem enquanto possibilidade de evidenciar a aplicação da Matemática em atividades do cotidiano.

Quanto à atividade de modelagem matemática, entendemos ser muito interessante, a qual ainda não conhecíamos, pois é possível criar situações e hipóteses voltadas ao cotidiano dos alunos, tendo em vista um dos motivos de desinteresse dos mesmos ser: para que estou aprendendo isso? Será que um dia usarei isso?

Fonte: Registro dos alunos

Outro grupo, G3, também destacou a importância da modelagem no ensino da Matemática, além de evidenciar o fato de que a aproximação dos modelos com a 
realidade depende dos conhecimentos matemáticos de que o estudante ou 0 pesquisador dispõe, conforme trecho destacado a seguir.

A adoção de modelos matemáticos seja na forma de apresentação, seja no processo de
criação é muito importante para os educandos que estudam mais variados níveis da
matemática, para que assim perceba a importância da matemática, suas linguagens,
relações e símbolos. [...] quanto maior é a proximidade do modelo com a realidade, mais
complexo será o modelo. Isto significa um maior número de parâmetros e
consequentemente uma maior dificuldade tanto na obtenção de dados a partir do modelo
quanto na interpretação desses dados gerados pelo modelo em questão.

Fonte: Registro dos alunos

Além dessa atividade, em 2017 também foi elaborada uma Produção Textual em Grupo para os estudantes do 6 - período do curso, envolvendo atividade de modelagem matemática com o tema $A$ doação de sangue no Brasil. Nessa proposta, os estudantes deveriam construir um modelo matemático que representasse os dados disponibilizados a eles nas orientações, os quais se referem ao percentual de doadores de sangue no Brasil em função das faixas etárias, dados obtidos a partir de pesquisa realizada sob o patrocínio da Agência Nacional de Vigilância Sanitária (ANVISA). Assim como na proposta de 2016, determinados grupos recorreram a representações gráficas para identificar o melhor modelo que representasse o conjunto de dados em questão, como o exemplo da Figura 1(b), apresentado pelo grupo G4. Devido à disposição dos pontos no plano, em geral, os modelos elaborados tomaram por base as funções polinomiais de $2^{\circ}$ grau.

Dentre as diferentes reflexões e comentários apresentados pelos estudantes, o grupo G5, conforme trecho apresentado a seguir, relatou a necessidade de buscar conhecimentos das diferentes disciplinas envolvidas na proposta, como a Modelagem Matemática e o Cálculo Diferencial e Integral, evidenciando a importância do papel ativo do estudante no desenvolvimento de atividades de modelagem, pois este precisa articular seus conhecimentos visando atingir um objetivo, que não consiste apenas na construção do modelo, mas também na interpretação dele e na construção dos demais conhecimentos matemáticos necessários para a realização da atividade.

Ao calcularmos passo a passo a atividade tivemos que recorrer a todo o material que nos
foi disponível bem como os livros de cálculo e modelagem em matemática, onde nos
auxiliou a alcançar nossos objetivos. 
Fonte: Registro dos alunos

Outro comentário apresentado pelo mesmo grupo G5 destacou, também, a importância do emprego de outras estratégias metodológicas para o ensino da Matemática na Educação Básica, dentre as quais se pode destacar a modelagem matemática, conforme trecho reproduzido a seguir.

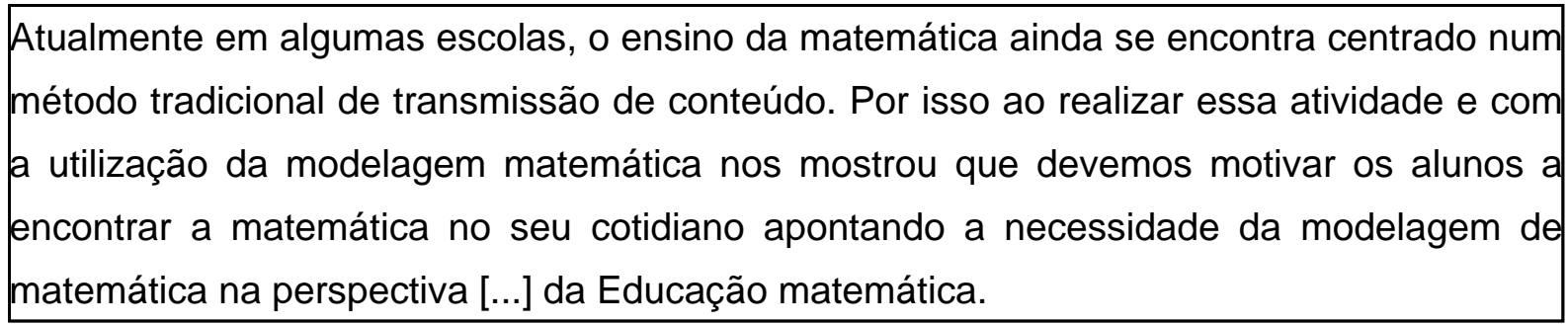

Fonte: Registro dos alunos

As diferentes propostas desenvolvidas, ao longo do curso de Licenciatura em Matemática, relacionadas à modelagem matemática, têm por objetivo proporcionar aos estudantes um contato com essa estratégia, além de um espaço para reflexões a respeito das potencialidades da modelagem matemática para o ensino de Matemática na Educação Básica.

\section{Considerações Finais}

A modelagem matemática, como apresentada neste trabalho, pode ser utilizada como uma alternativa pedagógica para o ensino de Matemática por meio de situações do cotidiano do estudante. Percebemos que as atividades de modelagem matemática, desenvolvidas ao longo do curso de Licenciatura em Matemática, na modalidade a distância, oportuniza ao estudante a construção de modelos matemáticos utilizando os conceitos matemáticos estudados ao longo do curso, além de permitir o desenvolvimento da habilidade de interpretar os resultados, visto que uma das etapas importantes da modelagem é a interpretação e a validação do modelo matemático, pois muitas vezes o modelo construído pode não se adequar à situação-problema. É importante ressaltar que a modelagem matemática pode ser desenvolvida para várias ciências, uma vez que convida os alunos a investigar situações variadas presentes nas ciências exatas, biológicas, etc. Nesse âmbito, a modelagem não se resume a manipular modelos matemáticos, mas permite ao aluno participar da elaboração deles, partindo de hipóteses e de leis que devem ser apreendidas. Por exemplo, um ensino de Física focado na modelagem permite que os alunos participem da criação dos modelos utilizados nos problemas, não encarando as leis físicas somente como relações entre 
grandezas. Indo mais além, permitindo uma discussão sobre a validade dos modelos com ênfase no fato de que eles precisam ser validados e, às vezes, refinados. $O$ foco será sempre o entendimento do fenômeno físico e não a resolução de problemas com aplicações de fórmulas.

\section{Referências}

ALMEIDA, L. M. W. de; DIAS, M. R. Um estudo sobre o uso da Modelagem Matemática como estratégia de ensino e aprendizagem. Bolema, Rio Claro - SP, v. 17, n. 22, 2004.

ALMEIDA, L. M. W., SILVA, K. P., \& VERTUAN, R. E. (2012). Modelagem Matemática na educação básica. São Paulo: Contexto.

BASSANEZI, R. C. Ensino-aprendizagem com modelagem matemática: uma nova estratégia. 3. Ed. São Paulo: Contexto Editora, 2011.

BORBA, M. de C.; MENEGHETTI, Renata Cristina Geromel; HERMINI, Helba Alexandra. Modelagem, calculadora gráfica e interdisciplinaridade na sala de aula de um curso de ciências biológicas. Revista de Educação Matemática da SBEM-SP, v. 5, n. 3, p. 63-70, 1997.

BURAK, D. Modelagem matemática: uma alternativa para o ensino de matemática na 5 a série. 1987. Dissertação (Mestrado em Educação Matemática)- Universidade Estadual Paulista Júlio de Mesquita Filho- Rio Claro, Rio Claro.

CALDEIRA, A. D. Modelagem Matemática: um outro olhar. Alexandria: Revista de Educação em Ciência e Tecnologia, 2(2), 33-54, 2009.

GIL, A. C. Métodos e Técnicas de Pesquisa Social. Atlas. São Paulo, 1999.

GOODE W.J, HATT P. K. Métodos em pesquisa social. 5a ed. São Paulo: Companhia Editora Nacional;1979.

MEYER, J. F. da C. de A.; CALDEIRA, A. D.; MALHEIROS, A. P. dos S. Modelagem em Educação Matemática. Belo Horizonte: Autêntica Editora, 2011. 